\title{
Immune Thrombocytopenic Purpura With Unusual Presentations - Reports of Two Cases
}

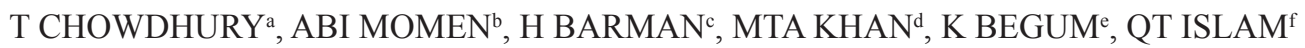

\begin{abstract}
:
Immune thrombocytopenic purpura (ITP) is an immune mediated bleeding disorder, usually has a relatively benign clinical course. Deep seated bleeding like intracranial haemorrhage or haemoperitonium or massive haemorrhage requiring transfusion or other intervention are rare in ITP, unless platelet count are extremely low or other complicating conditions coexist. Here are two case reports of ITP presenting in uncommon and devastating manners. The $1^{\text {st }}$ one is of a 21-yearold married nulliparaous lady with ITP complicating her undiagnosed ovarian hyperstimulation syndrome leading to haemoperitonium (ruptured ovarian cyst), post operative alveolar haemorrhage resulting in ARDS and later on DVT of right leg on her $9^{\text {th }}$ POD. She was managed by multi
\end{abstract}

Introduction:

Idiopathic thrombocytopenic purpura (ITP) is mediated by autoantibodies, most often directed against the platelet membrane glycoprotein IIb/IIIa, which sensitize the platelet, resulting in premature removal from the circulation by cells of the reticulo-endothelial system. It is not a single disorder; some cases occur in isolation while others are associated with underlying immune dysregulation in conditions such as connective tissue diseases, HIV

a. Tasmina Chowdhury, Registrar of Medicine, Popular Medical College.

b. Abdul Basit Ibne Momen, Registrar of Medicine, Bangladesh Medical College.

c. Hironmoy Barman, Assistant registrar of medicine, Popular Medical College

d. Mohammad Tariqul Ahsan Khan, Assistant registrar of medicine, Popular Medical College.

e. Kohinoor Begum, Prof. of Gynaecology, Popular Medical College

f. Quazi Tarikul Islam Professor and Head, Dept of Medicine, Popular Medical College.

Address of correspondence: Dr. Tasmina Chowdhury, FCPS (Medicine), Registrar of medicine, Popular medical college, email: tasminac7@gmail.com.

Received: 27 October, 2019

Accepted: 07 September, 2020 discipline team. A new consequence of her disease one after another was striking and made her management more challenging. Ultimately the lady recovered and was discharged with advice which was not less than a miracle. The $2^{\text {nd }}$ case is of a 50- year- old elderly lady who had a hemorrhagic stroke as a presenting feature of ITP. Though ITP is not an uncommon disease but in these cases its presentation, consequences and severity was unusual and making its management very much challenging.

Key words: ITP, ovarian hyperstimulation syndrome, haemorrhage, Stroke

(J Bangladesh Coll Phys Surg 2020; 38: 218-222)

DOI: https://doi.org/10.3329/jbcps.v38i4.48983

infection, B cell malignancies, pregnancy and certain drug therapies. However, the clinical presentation and pathogenesis are similar, whatever the cause of ITP.

Patient usually presents with superficial bleeding like purpura, gum or nose bleeding, menorrhagia sometimes haematuria or bloody stool. Deep seated bleeding like intracranial haemorrhage or haemoperitonium is rare with ITP.

The presentation depends on the degree of thrombocytopenia. Spontaneous bleeding typically occurs only when the platelet count is below $20 \times$ $10^{9} / \mathrm{L}^{1}$. At higher counts, the patient may complain of easy bruising or sometimes epistaxis or menorrhagia. Many cases with counts of more than $50 \times 10^{9} / \mathrm{L}$ are discovered by chance. In adults, ITP more commonly affects females and may have an insidious onset. ITP in children is usually acute with history of a preceding viral infection but in most adult cases it tends to be chronic (persisting for more than 1 year) ${ }^{2}$. Symptoms or signs of a connective tissue disease 
may be apparent at presentation or emerge several years later. The peripheral blood film is normal, apart from a greatly reduced platelet number, whilst the bone marrow reveals an obvious increase in megakaryocytes.

\section{CASE 1}

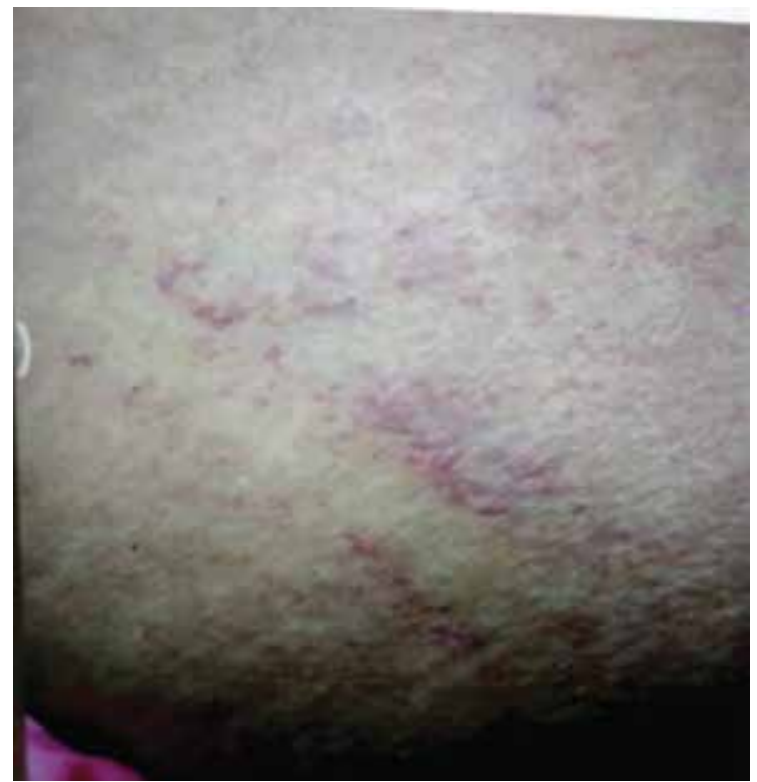

Fig: 1 purpuric spots on skin

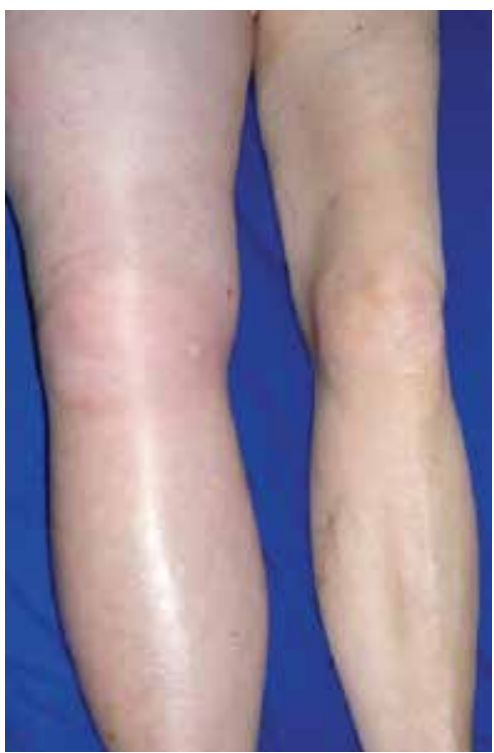

Fig: 2 DVT of right leg

A 21-year-old lady, presented to the emergency with the complains of reddish spots all over the body (Fig: $1)$, specially on neck and extremities of multiple size for the last 3 days with occasional bleeding from the nose and gum for the same duration. She also complained that she had been suffering from on and off abdominal pain for last 3 weeks, pain started from left lower abdomen which was dull in nature, mild to moderate in intensity. Two days after the start of abdominal pain, patient started menstruating but this time the bleeding was very severe and persisted for almost 10 days. Her drug history revealed she had been taking ovulation inducing drug Letrozole as she was on treatment for subfertility.

Physical examination revealed, the patient was moderately anaemic ,moderately dehydrated. Reddish purple purpura was present over the extremities which were palpable, non-pruritic, and did not blanch on pressure. She was tachypnoeic (24 breaths/min), had tachycardia(120 beats/min), with normal blood pressure, temperature. She had no bony tenderness or lymhadenopathy. Abdomen was distended, tense, and shiny with full flanks, everted umbilicus. There was presence of shifting dullness. Few fine crepitation were present at base of both lungs.

Lab reports revealed that haemoglobin was $4.1 \mathrm{~g} / \mathrm{dl}$, platelet 5,000 , WBC 7,370, $\beta$-HCG was negative, normal coagulation profile.ANA was negative.

With a high suspicion of ITP patient was put on high dose $\mathrm{i} / \mathrm{v}$ methylprednisolone along with blood transfusion as bone marrow confirmation was not possible at that time due to patient condition.

The next day after admission, her abdominal pain became severe and was spasmodic initially, later becoming continuous in nature along with gradual abdominal distension. Along with these she developed chest tightness and breathlessness and was becoming paler.

An urgent ultrasonogram revealed gross haemoperitoneum (about $2000 \mathrm{ml}$ ) with echogenic debris floating inside the Pouch of Douglas. The uterus was non gravid. There was a lesion in left tubo ovarian region (?endometrioma/ haematoma).

Consultation was sought immediately form the Gynaecology department, who advised to do exploratory laparotomy. In the meantime the patient had received four bags of fresh whole blood and 3 bags of apheretic platelet in order to prepare her for surgery

After opening the abdomen, the left ovary was enlarged about $12 \times 10 \mathrm{~cm}$ with multiple ruptured 
cysts all over the cortical surface with hematoma in cyst cavity. It was also adherent with posterior wall of uterus. The left tube was edematous and looked unhealthy. Patient received 5 units of whole blood transfusion and 1 unit of aphaeretic platelet transfusion during the operation.

After the surgery she was on oral steroid and eltrombopag on the clinical suspicion of ITP, with appropriate antibiotic coverage.

Shortly after transferring the patient to the post operative ward she developed severe respiratory distress and had to be shifted to the intensive care unit where her blood gas revealed mixed acidosis and her oxygen saturation was falling which led to her being intubated during which time profuse amount of blood came out while giving suction due to alveolar haemorrage. She gradually recovered and was extubated after three days.

Her platelet count showed not satisfactory improvement, ranging between 20,000 to 30,000 .

Nine days after the operation while she was recovering otherwise she developed severe pain and swelling of her right leg (Fig: 2). On examination the right leg looked reddish and tense than left leg, local temperature was increased, tenderness present, bulk of the muscle increased on right leg. Peripheral pulses on both legs were present. FDP and D-Dimer showed increased value. Duplex study of lower limb vessels revealed right external iliac, common femoral, superficial femoral and popliteal venous thrombosis without sign of recanalization. Markers of prothrombotic states (Homocystine, Protein C, Protein S, anti-phospholipid antibody) were negative.ANA was repeatedly negative. Low molecular weight heparin was started considering benefit over risk, and compression stocking was advised for use.

Once the patient was stable bone marrow examination confirmed immune thrombocytopenic purpura. After 4 weeks of trial with steroid, mycophenolate mofetil was added as there was little benefit from steroid. Then her platelet count showed gradual improvement.

So, the final diagnosis was Immune Thrombocytopenic Purpura, Ovarian Hyperstimulation Syndrome with ruptured Ovarian Cyst leading to Haemoperitonium, Alveolar Hemorrhage causing Acute Respiratory Distress Syndrome (ARDS), Deep Vein Thrombosis of right lower limb.
CASE 2

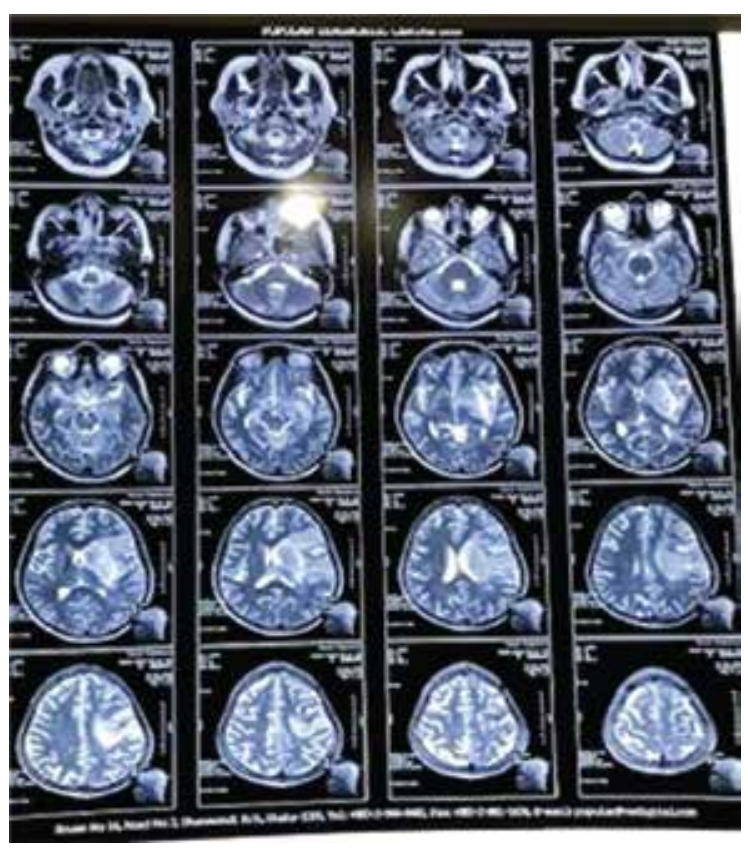

Fig-3: MRI brain showing subacute intracerebral hematoma within the left lentiform nucleus with perilesional edema causing midline shift.

A 50-year-old normotensive non-diabetic lady was brought to the emergency department with complains of sudden onset weakness of right side of the body for 10 days. Her attendant stated that after a sudden fall she was unable to move right side of her body. The weakness started from patient's right upper limb then gradually involved the lower limb as well leaving her unable to walk. She had stopped talking, was unable to follow command and gradually became drowsy within the last 5 days. Apart from her age she did not have any risk factor for stroke. Her attendant mentioned that they noticed multiple reddish spots on her skin which initially appeared over the arms and legs later involved the other parts of the body in the last 1 month. The spots were initially red then turned blue and then black after a few days. The patient also had multiple episodes of gum bleeding in the last 1 month.

On examination the patient was disoriented, had motor aphasia with GCS 11, anaemic, regular pulse, right sided hemiparesis with extensor planter response. However, there were multiple purpuric spots on different parts of the body, of varying sizes, painless and did not blanch on pressure. 
Initial clinical suspicion was haemorrhagic stroke due to Aplastic anaemia or Acute leukaemia.

Full blood count on admission revealed- total WBC was 17,000 with $75 \%$ neutrophils, $\mathrm{Hb} 10 \mathrm{~g} / \mathrm{dL}$ and platelet only 15,000. Peripheral blood film showed marked thrombocytopenia, hypochromic microcytic anaemia and leukocytosis. Repeat platelet 3 days later was 30,000. Urine routine showed plenty RBC and 12-16 pus cells and later urine culture was positive for E. coli. MRI brain(fig-3) revealed subacute intracerebral haematoma $(42 \times 20 \times 28 \mathrm{~mm})$ within the left lentiform nucleus with perilesional edema causing midline shift. Anti-nuclear factor (ANA) and Coombs test were negative. Bone marrow examination was consistent with Immune thrombocytopenic purpura.

She was put on steroid and regular physiotherapy with other supportive care of stroke. Gradually she regained orientation and her motor functions were slightly improved. She was discharged with advice.

\section{Discussion:}

No firm data are available on the natural history of ITP or on mortality rates or frequency of major bleeding episodes associated with this condition. The disease thought to have a relatively benign course, despite the occurrence of vey low platelet count. The American society of Haematology (ASH) ITP guideline panel recently published their conclusions based on a systemic review of clinical series. They estimated that the risk of fatal haemorrhage was $5 \%$ throughout the lifetime of a patient with $\mathrm{ITP}^{3}$.The estimate refers to the patients with platelet count below $30 \mathrm{X} 10^{9} / \mathrm{L}$, a level commonly accepted as a threshold for the occurrence of major bleeding among the ITP patients. Our first patient was taking Letrozole (oral anti-estrogen with ovulation inducing property) for her subfertility and developed OHSS which remained undiagnosed. Concomitantly she developed ITP which complicated her OHSS leading to grievous complication like haemoperitonium. Ovarian hyperstimulation syndrome is an iatrogenic complication of assisted reproduction technology. The syndrome is characterized by cystic enlargement of ovaries and a fluid shift from the intravascular to third space due to increased capillary permeability and ovarian neoangiogenesis. Enlargement of ovaries causes abdominal pain, nausea, leakage of fluid from follicles, increased capillary permeability leading to third spacing (due to release of vasoactive substances) or frank rupture of follicle can all cause ascites $^{4}$. In our case OHSS was diagnosed on the basis of multiple large cysts on laparotomy, with a history of ovulation inducing drug followed by abdominal pain. However postoperative massive alveolar haemorrhage on next day which occurred due to intubation during anaesthetic procedure with low platelet count, leading to ARDS was another striking event. Patient also recovered from this situation but complications hadn't completed its journey yet. On her $9^{\text {th }}$ POD while recovering otherwise, she developed DVT of right leg. Thromboembolic events have been reported in up to $8 \%$ of patients with ITP $^{5}$. Thromboembolism can occur with the introduction of ITP therapies like steroid $^{6-8}$, splenectomy ${ }^{9}$ and thrombopoietin receptor agonists $^{10-12}$.DVT can also occur as a complication of $\mathrm{OHSS}^{13}$.However the actual cause of DVT was not certain and common hypercoagulable states were excluded. Starting anticoagulant in such a patient with low platelet count was another challenge. Meanwhile her ITP was also nonresponsive to steroid and eltrombopag making management more difficult. But ultimately with this management her DVT subsided without any further complication and platelet count was improved with the addition of MMF.

Haemorrhagic stroke is another uncommon complication of ITP that happened in our $2^{\text {nd }}$ case. The learning from these two cases was ITP is not always as benign as we get in our day to day practice. But early diagnosis, prompt and proper management can save patient's life.

\section{References:}

1. HG Watson, DJ Culligan, LM Manson. (2018).Davidson's principle and practice of medicine.(23 $3^{\text {rd }}$ edition).

2. http// Journal List .Int J Gen Med. v.3; 2010. PMC2962326/Long-term management of chronic immune thrombocytopenic purpura in adults

3. Yael C,Cohen, Benjamin Djulbegovic, Orna ShamaiLubivitz, Benjamin Mozes. (Rerinted)ARCH INTERN MED/VOL 160,June 12 2000.Downloaded from https://jamanetwork.com on06/23/2019.

4. http//www.ncbi.nim.nih.gov>pmc.

5. Takagi S, Suzuki I, Watanabe S (2015) Risk of Thromboembolism in Patients with Immune 
Thrombocytopenia. J Hematol Thrombo Dis 2015,3:1.

6. Huerta C, Johansson S, Wallander MA, García Rodríguez LA (2007) Risk factors and short-term mortality of venous thromboembolism diagnosed in the primary care setting in the United Kingdom. Arch Intern Med 167: 935-943.

7. Johannesdottir SA, Horváth-Puhó E, Dekkers OM, Cannegieter SC, Jørgensen JO, et al. (2013) Use of glucocorticoids and risk of venous thromboembolism: a nationwide population-based case-control study. JAMA Intern Med 173: 743-752.

8. Stuijver DJ Majoor CJ, van Zaane B, Souverein PC, de Boer A, et al. (2013) Use of oral glucocorticoids and the risk of pulmonary embolism: a population-based case-control study. Chest 143: 1337-1342.
9. Vianelli N, Palandri F, Polverelli N, Stasi R, Joelsson J, et al. (2013) Splenectomy as a curative treatment for immune thrombocytopenia: a retrospective analysis of 233 patients with a minimum follow up of 10 years. Haematologica 98: 875-880.

10. Provan D, Stasi R, Newland AC, Blanchette VS, Bolton-Maggs P, et al.(2010) International consensus report on the investigation andmanagement of primary immune thrombocytopenia. Blood 115:168-186

11. Neunert C, Lim W, Crowther M, Cohen A, Solberg L Jr, et al. (2011) TheAmerican Society of Hematology 2011 evidence-based practice guidelinefor immune thrombocytopenia. Blood 117: 4190-4207.

12. Lakshmanan S, Cuker A (2012) Contemporary management of primaryimmune thrombocytopenia in adults. J Thromb Haemost 10: 1988-1998

13. http://www.myoclinic.org $>$ syc2 